\title{
Metamaterial-Based Zeroth-Order Resonant Antennas for MIMO Applications
}

\author{
Gunyoung Kim $\cdot$ Bomson Lee*
}

\begin{abstract}
A compact $\left(0.26 \times 0.05 \lambda_{0}\right.$ at $\left.2.27 \mathrm{GHz}\right)$ metamaterial-based zeroth-order resonant antenna system consisting of epsilon-negative (ENG) and mu-negative (MNG) structures is proposed. Although the spacing between the ENG and MNG antennas is only $0.09 \lambda_{0}$, the isolation is relatively high $(27.6 \mathrm{~dB}$ at $2.27 \mathrm{GHz})$. Furthermore, the envelope correlation is only 0.015 . The radiation efficiencies of the proposed two radiators are also very high ( $90 \%$ on average).

Key Words: Epsilon Negative, Metamaterial, MIMO, Mu Negative, Zeroth-Order Resonant.
\end{abstract}

\section{Introduction}

Multiple-input multiple-output (MIMO) technology is now widely employed in order to increase the reliability and the channel capacity of modern wireless communication systems. However, the available space for antennas in recent mobile terminals is very limited. Moreover, meeting the requirements of compact size, high isolation, low envelope correlation, and high radiation efficiencies in limited space usually available is quite challenging $[1,2]$. To achieve these goals, many antenna structures have been proposed. In [3], a planar inverted-F antenna (PIFA) with a dual-feed was presented. Two PIFAs are placed at the corners of a mobile terminal for the largest possible separation, utilizing polarization diversity [4]. There have been trials to use photonic band-gap or split ring resonator [5] structures between the radiation elements in order to improve the isolation. A decoupling network [6] is one promising means of satisfying the MIMO requirements. However, these two methods require an additional structure or circuit between the antennas. To overcome this problem, we adopt two types of metamaterial (MTM) zeroth-order resonant (ZOR) antennas. One is the epsilon-negative (ENG; open terminated) antenna, and the other is the mu-negative (MNG; short terminated) antenna [7]. It is well known that MTM ZOR antennas can be made as small as possible. In addition, it will be shown that the performance of the proposed MIMO system far surpass those of recent works in terms of the size, isolation, envelope correlation, and antenna efficiencies.

\section{II . Antenna Design and Simulation}

Fig. 1 shows the geometry of the proposed MIMO system composed of the ZOR ENG and MNG antennas with their dimensions designed at $2.2 \mathrm{GHz}$. The antennas are fed by two $50 \Omega$ microstrip lines with width of $7.5 \mathrm{~mm}$ on a Teflon substrate $\left(\varepsilon_{r}, 2.2\right.$; height, 2.37 $\mathrm{mm})$. Since the ENG antenna has an open termination and its propagation constant $\beta$ is zero (zeroth-order), the series current is negligible and the shunt voltage wave (or electrical field) is dominant in the entire structure with the same phase. For the MNG antenna with a

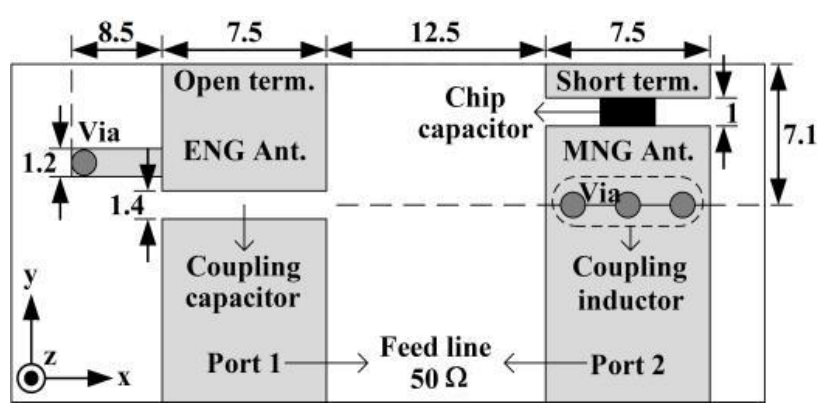

Fig. 1. Geometry of proposed multiple-input multiple-output system consisting of epsilon-negative (ENG) and mu-negative (MNG) antennas (unit: $\mathrm{mm}$ ).

Manuscript received May 30, 2013 ; Revised June 27, 2013 ; Accepted August 1, 2013. (ID No. 20130530-027J)

Department of Electronics \& Radio Engineering, Kyung Hee University, Yongin, Korea.

*Corresponding Author : Bomson Lee (e-mail : bomson@khu.ac.kr)

This is an Open-Access article distributed under the terms of the Creative Commons Attribution Non-Commercial License (http://creativecommons.org/licenses/ by-nc/3.0) which permits unrestricted non-commercial use, distribution, and reproduction in any medium, provided the original work is properly cited. 
short termination, the opposite is true with a dominant series current. The electric fields generated by this current are orthogonal to those in the ENG antenna. For this reason, the coupling between the two antenna elements becomes very small.

In Fig. 2, we show the equivalent circuit of the MNG antenna short-terminated. The electrical length $k d$ of the host transmission line is $\pi / 6$ radians at $2.4 \mathrm{GHz}$ and the characteristic impedance $Z_{c}$ is $50 \Omega$. This is equivalent to the fact that the series inductance $L d$ is $1.74 \mathrm{nH}$ and the shunt capacitance $C d$ is $0.69 \mathrm{pF}$. Since the propagation constant $\beta$ is required to be 0 at the transient frequency of $f_{0}=2.4 \mathrm{GHz}, C_{0}$ is determined to be $2.5 \mathrm{pF}$ [7]. Furthermore, $R_{0}$ is extracted to be $0.23 \Omega$. Since $R_{0}$ is very small and direct matching to the feed line is not possible, a coupling inductor with $L_{1}$ is usually required. By equating the input admittance $Y_{\text {in }}$ to the characteristic admittance $Y_{c}$ of the feed line, we can find a new resonant angular frequency $\omega_{1}$ given by

$$
\omega_{1}=\omega_{0}-\frac{1}{2 L d} \cdot \sqrt{R_{0}\left(Z_{C}-R_{0}\right)}
$$

and $L_{1}$ is found to be

$$
L_{1}=\frac{1}{\omega_{1}} \cdot \frac{Z_{C} R_{0}}{\sqrt{R_{0}\left(Z_{C}-R_{0}\right)}+\omega_{1}(C d) Z_{C} R_{0}} .
$$

In (1), as $R_{0} \rightarrow Z_{c}$ or $0, \omega_{1} \rightarrow \omega_{0}$, and $\omega_{1}$ becomes the lowest when $R_{0}$ is $Z_{c} / 2$. Using (1) and (2), the new resonant frequency $f_{1}$ becomes $2.23 \mathrm{GHz}$ and $L_{1}$ is calculated to be $0.23 \mathrm{nH}$, realized using three vias with radius $0.5 \mathrm{~mm}$ as seen in Fig. 1 .

For the ENG, the equivalent circuit is exactly the dual of Fig. 2 [8]. The calculated shunt inductance $L_{0}$ is 6.2 $\mathrm{nH}$, realized with a shorted stub, and $G_{0}$ is extracted to be $0.0001 \mathrm{~S}$. The coupling capacitance $C_{1}$ for matching to the feed line is $0.1 \mathrm{pF}$, realized with a gap, as seen in Fig. 1. The overall MIMO antenna system is very compact $\left(0.26 \times 0.05 \quad \lambda_{0}\right)$.

Fig. 3 shows the photograph of the MIMO system consisting of ENG and MNG antennas. Two feed lines have been somewhat bent away from each other for easy mounting of SMA connectors. Fig. 4(a) and (b) show the electromagnetic (EM)-simulated and measured

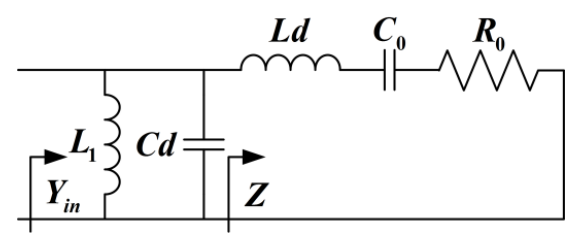

Fig. 2. Equivalent circuit for the short-terminated mu-negative antenna.

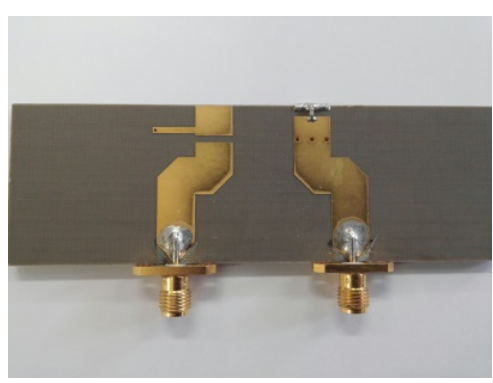

Fig. 3. Fabricated multiple-input multiple-output system.

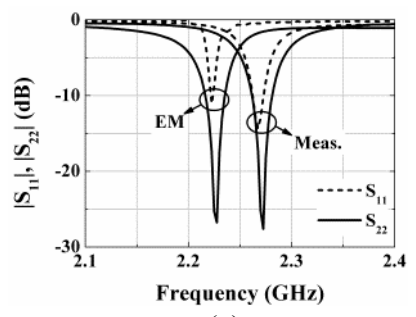

(a)

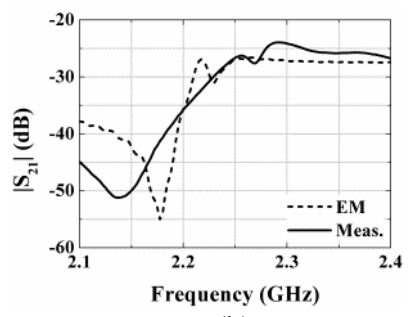

(b)
Fig. 4. Electromagnetic (EM)-simulated and measured $S$ parameters of the proposed antenna: (a) $\left|S_{11}\right|,\left|S_{22}\right|$ and (b) $\left|S_{21}\right|$.

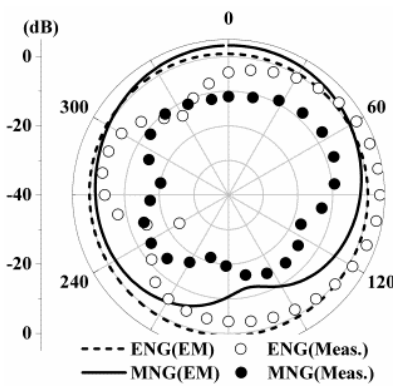

(a)

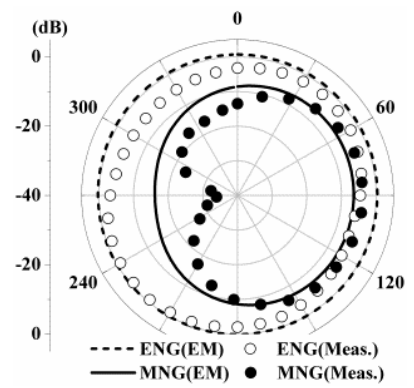

(b)
Fig. 5. Electromagnetic (EM)-simulated and measured gain patterns in yz plane: (a) $E_{\theta}$ and (b) $E_{\phi} . \quad E N G=e p-$ silon-negative, $\mathrm{MNG}=\mathrm{mu}$-negative.

$\left|S_{11}\right|$ (ENG), $\left|S_{22}\right|(\mathrm{MNG})$, and $\left|S_{21}\right|$. Their resonant frequencies are shown to be off by about $0.04 \mathrm{GHz}$, possibly due to fabrication inaccuracy. The EM-simulated and measured resonant frequencies are 2.23 and $2.27 \mathrm{GHz}$, respectively. Although the spacing between the ENG and MNG is only $0.09 \lambda_{0}$, the isolation $\left(\left|S_{21}\right|\right)$ which has been measured using a network analyzer is relatively high $(27.6 \mathrm{~dB})$ at $2.27 \mathrm{GHz}$.

In Fig. 5(a) and (b), we show the gain patterns $E_{\theta}$ and $E_{\phi}$ of the ENG and MNG antennas, respectively, in the yz plane. The EM-simulated and measured patterns of $E_{\phi}$ are in a better agreement than those of $E_{\theta}$. The difference between the EM-simulated and measured pattern of $E_{\theta}$ is believed to come from the fabrication error. Due to the small size of the antennas and ground plane, they show near-isotropic patterns. The envelope 
Table 1. Comparison of multiple-input multiple-output antenna performances

\begin{tabular}{|c|c|c|c|c|}
\hline \multirow{2}{*}{ Criteria } & A type [4] & B type [5] & C type [6] & Proposed \\
\hline & Port 1 & Port 1 & Port 1 & Port 1 \\
\hline Antenna size & $0.5 \times 0.25 \quad \lambda_{0}$ & $0.125 \times 0.77 \quad \lambda_{0}$ & $0.18 \times 0.07 \quad \lambda_{0}$ & $0.26 \times 0.05 \quad \lambda_{0}$ \\
\hline Antenna spacing & $0.5 \lambda_{0}$ & $0.125 \lambda_{0}$ & $0.09 \lambda_{0}$ & $0.09 \lambda_{0}$ \\
\hline$f_{R}(\mathrm{GHz})$ & 3 & 1.24 & 2.45 & 2.27 \\
\hline$\left|S_{11}\right|,\left|S_{21}\right|(\mathrm{dB})$ & Near $-25,-16.5$ & $\begin{array}{l}\text { Near }-11 \text {, } \\
\text { Below }-40\end{array}$ & $-27,-20$ & $-14.4,-27.6$ \\
\hline$\rho_{e}($ envelope correlation $)$ & Not mentioned & Below 0.1 & Not mentioned & 0.015 \\
\hline Gain $(\mathrm{dBi})$ & 4.85 & 5.6 & 1.57 & 2.8 \\
\hline Radiation efficiency (\%) & Not mentioned & 60 & Not mentioned & 97 \\
\hline
\end{tabular}

correlation [9] of the proposed antenna has been computed to be very small $(0.015)$. This result is due to the near-orthogonally radiated fields, evaluated over all $4 \pi$ solid angles.

In Table 1, the various performances of the proposed antennas are compared with those in [4] (A type), [5] (B type), and [6] ( $\mathrm{C}$ type), and the proposed antennas prove to be superior to the others to the best of our knowledge. The gains and radiation efficiencies of the each antenna were measured while the one port was connected to the source and the other one was matched.

\section{Conclusion}

A very compact MTM-based ZOR MIMO system consisting of ENG and MNG structures has been designed, fabricated, and evaluated in terms of its size, isolation, envelope correlation, and radiation efficiencies. The performances of the proposed antenna have been compared with those of recent competitive works. Despite the small separation $\left(0.09 \lambda_{0}\right)$ between the proposed radiation elements, the isolation is relatively high $(27.6 \mathrm{~dB})$ at $2.27 \mathrm{GHz}$. The envelope correlation has been found to be negligibly small (0.015). The efficiencies are about $90 \%$ on average. The proposed ZOR MIMO antenna system is considered to be an immediate candidate for the very compact mobile handsets of today.

This work was supported by the Mid-career Researcher Program through the National Research Foundation of Korea (NRF) grant funded by the Korea government (No. 2012047938).

\section{References}

[1] T. Svantesson, "Correlation and channel capacity of MIMO systems employing multimode antennas," IEEE Transactions on Vehicular Technology, vol. 51, no. 6, pp. 1304-1312, Nov. 2002.

[2] P. S. Kildal and K. Rosengren, "Correlation and capacity of MIMO systems and mutual coupling, radiation efficiency, and diversity gain of their antennas: simulations and measurements in a reverberation chamber," IEEE Communications Magazine, vol. 42, no. 12, pp. 104-112, Dec. 2004.

[3] H. T. Chattha, Y. Huang, S. J. Boyes, and X. Zhu, "Polarization and pattern diversity-based dual-feed planar inverted-F antenna," IEEE Transactions on Antennas Propagation, vol. 60, no. 3, pp. 1532-1539, Mar. 2012.

[4] M. K. T. Al-Nuaimi and W. G. Whittow, "Performance investigation of a dual element IFA array at $3 \mathrm{GHz}$ for MIMO terminals," in Proceedings of the Loughborough Antennas \& Propagation Conference, Loughborough, UK, 2011, pp. 1-5.

[5] M. M. Bait-Suwailam, M. S. Boybay, and O, M. Ramahi, "Electromagnetic coupling reduction in highprofile monopole antennas using single-negative magnetic metamaterials for MIMO applications," IEEE Transactions on Antennas Propagation, vol. 58, no. 9, pp. 2894-2902, Sep. 2010.

[6] S. C. Chen, Y. S. Wang, and S. J. Chung, "A decoupling technique for increasing the port isolation between two strongly coupled antennas," IEEE Transactions on Antennas and Propagation, vol. 56, no. 12, pp. 3650-3658, Dec. 2008.

[7] S. Jang and B. Lee, "Modelling and design of resonant one-dimensional metamaterial-based transmission lines for antenna applications," IET Microwaves Antennas \& Propagation, vol. 5, no. 13, pp. 15291536, Oct. 2011.

[8] T. G. Kim and B. Lee, "Metamaterial-based compact zeroth-order resonant antenna," Electronics Letters, vol. 45, no. 1, pp. 12-13, Jan. 2009.

[9] S. Blanch, J. Romeu, and I. Cordella, "Exact representation of antenna system diversity performance from input parameter description," Electronics Letters, vol. 39, no. 9, pp. 705-707, May 2003. 